Rev. Int. Contam. Ambie. 32 (3) 255-266, 2016

DOI: 10.20937/RICA.2016.32.03.01

\title{
TRATAMIENTO DE VINAZAS EN UN REACTOR DE LECHO FLUIDIZADO INVERSO ANAEROBIO
}

\author{
Eric HOUBRON ${ }^{1}$, Martha Elvira SANDOVAL ROJAS ${ }^{2 *}$ y Aurelio Felix HERNÁNDEZ MUÑOZ ${ }^{3}$
}

${ }^{1}$ Facultad de Ciencias Químicas de Orizaba, Universidad Veracruzana. Prolongación Avenida Oriente 6, 1009, Colonia Centro, Orizaba, Veracruz, México, C.P. 94340

${ }^{2}$ Unidad de Investigación del Medio Ambiente, Tecnológico Nacional de México, Instituto Tecnológico de Minatitlán. Boulevard Institutos Tecnológicos s/n. Colonia Buena Vista, Norte, Minatitlán, Veracruz, México, C.P. 96848

${ }^{3}$ Escuela Técnica Superior de Ingenieros de Caminos Canales y Puertos, Universidad Politécnica de Madrid. C/Profesor Aranguren s/n, Madrid, España, 28040

*Autor para correspondencia: marsanrojas@hotmail.com

(Recibido abril 2015; aceptado noviembre 2015)

Palabras clave: efluentes de producción de etanol, digestión anaerobia, carga orgánica volumétrica, tiempo de residencia hidráulica

\section{RESUMEN}

En la producción de etanol se generan vinazas, efluentes con $\mathrm{pH}$ ácido y elevada demanda química de oxígeno (DQO). Los sistemas de tratamiento anaerobio con biopelícula son una tecnología consolidada para tratamiento de efluentes industriales. El reactor de lecho fluidizado inverso anaerobio (LFI), empleado en el tratamiento de efluentes de alta carga, utiliza un soporte que proporciona una gran superficie y un bajo requerimiento de energía para la fluidización del lecho. Este trabajo evalúa el desempeño de un LFI, empleando Extendospher ${ }^{\mathbb{B}}$ como soporte y tratando efluentes de la producción de etanol. El reactor arrancó por lotes, posteriormente operó en continuo a diferentes cargas orgánicas volumétricas: $0.5,1.0,3.3,6.8$ y $10.4 \mathrm{gDQO} / \mathrm{L} \mathrm{d}$. Además, se evaluaron diferentes tiempos de residencia hidráulica (TRH): 10, 5 y 1 días. El sistema alcanzó las siguientes eficiencias promedio de remoción de DQO: $81 \%$ para la operación en lotes, y $58,67,59$ y $50 \%$ con cargas de $0.5,1.0,3.3,6.8$ $\mathrm{gDQO} / \mathrm{L}$ d respectivamente. Para la carga de $10.4 \mathrm{gDQO} / \mathrm{L} \mathrm{d}$, la eficiencia promedio de remoción de DQO fue $38 \%$, en esta condición el reactor presentó inestabilidad y disminución del rendimiento de metano. La generación de metano inició hasta los 110 días de operación del reactor a una carga de $1.0 \mathrm{gDQO} / \mathrm{L}$ d. El sistema alcanzó un rendimiento de metano desde 0.15 hasta $0.34 \mathrm{LCH}_{4} / \mathrm{gDQO}_{\text {removida. }}$. El reactor operando a una carga constante de $6.4 \mathrm{gDQO} / \mathrm{L} \mathrm{d}$, y TRH de 1 día, alcanzó una eficiencia promedio de remoción de DQO de $52 \%$.

Key words: effluents of ethanol production, anaerobic digestion, organic load rate, hydraulic retention times

\begin{abstract}
ABSTRAC
The vinasses are the residual liquid waste after distillation of the ethanol. This distillery effluent generally has a very high chemical oxygen demand (COD) concentration and
\end{abstract}


low $\mathrm{pH}$. Anaerobic treatment whit biofilms systems is a well-established technology for the treatment of industrial effluents. The anaerobic inverse fluidized bed reactor (IFBR) has been developed to provide biological treatment of high strength organic wastewater for their large specific surface and their low energy requirements for fluidization. This study describes the performance of IFBR with Extendospher ${ }^{\circledR}$, for the treatment of vinasses. The start-up was in batch, increasing gradually the organic load rate (OLR): 0.5 , $1.0,3.3,6.8$ and $10.4 \mathrm{gCOD} / \mathrm{L} \mathrm{d}$. Different hydraulic retention times (HRT) were evaluated: 10,5 and 1 days. During the batch operation, the COD removal obtained was of $81 \%$, and for OLR of $0.5,1.0,3.3,6.8 \mathrm{gCOD} / \mathrm{L} d$ the removal obtained was $58,67,59$ and $50 \%$ respectively. For a maximum OLR of $10.4 \mathrm{gCOD} / \mathrm{L} \mathrm{d}$, the COD removal was $38 \%$, and the system presented instability and a decrease of yield methane. The methane production initiated after 110 days from the start-up of the IFBR, to organic load rate of $1.0 \mathrm{gCOD} / \mathrm{L}$ d. The system reached values in the methane yield from $0.15 \mathrm{up}$ to $0.34 \mathrm{LCH}_{4} / \mathrm{g} \mathrm{COD}_{\text {removed, }}$ for the different organic load rates. During the operation to a constant OLR of $6.4 \mathrm{gCOD} / \mathrm{L}$ d, and a HRT of 1 day, the IFBR reached a maximum efficiency of removal of $52 \%$.

\section{INTRODUCCIÓN}

Los combustibles fósiles, el petróleo y sus derivados, como fuentes de energía no renovable, están llegando a niveles de agotamiento acelerado. Esta situación, sumada al calentamiento global provocado en gran medida por el uso de este tipo de combustibles, es una oportunidad para la implementación de energías renovables. En este sentido, la producción de biocombustibles ha despertado el interés mundial. Además de ser empleado como biocombustible, el etanol obtenido a partir de melaza de azúcar de caña, es utilizado para producir bebidas. En México, diversos ingenios poseen destilerías con una capacidad instalada para producir anualmente cerca de $167 \mathrm{mil}$ $\mathrm{m}^{3}$ de etanol. Independientemente del uso que se dé a éste, es importante considerar que las grandes cantidades producidas a nivel mundial (55 700 millones de L en 2007, empleados $30 \%$ en la industria, $9 \%$ en bebidas y $61 \%$ como combustibles) generan a su vez considerables volúmenes de efluentes de la destilación, denominados vinazas (COLPOS 2010). Estudios realizados en diversas destilerías indican que por cada litro de alcohol destilado se producen de 12 a 15 L de vinazas (Jiménez et al. 2006, Travieso et al. 2006, Lorenzo et al. 2015). Estas vinazas tienen un pH ácido (de 3 a 4). Asimismo, están caracterizadas por su alta demanda química de oxígeno (DQO) en un rango de 50-100 g DQO/L, dependiendo del origen del sustrato empleado en la fermentación alcohólica (Jiménez et al. 2006, Travieso et al. 2006).

Las vinazas con frecuencia han sido tratadas mediante procesos anaerobios. El tratamiento anaerobio de efluentes con elevado contenido de materia orgánica biodegradable presenta diversas ventajas, por ejemplo se puede alcanzar alta eficiencia de remoción alimentando altas cargas, presenta bajos requerimientos de nutrientes, genera pequeñas cantidades de lodos y produce un biogás combustible. La producción de biogás permite al proceso no sólo ahorrar energía, sino también generarla. Esto puede reducir significativamente los costos de operación comparado con el alto consumo de energía de los procesos aerobios (Fernández et al. 2008). Sin embargo, uno de los mayores problemas en los procesos anaerobios de tratamiento de aguas residuales es la pérdida de biomasa en sistemas con alta carga hidráulica. En relación con esto, muchos de los sistemas anaerobios empleados involucran sistemas de biomasa fija. La biopelícula proporciona interesantes ventajas tales como: ofrece una mayor superficie de contacto entre la materia orgánica y la biomasa, además de la fácil separación del agua tratada de la biomasa y de un diseño de sistema compacto (Houbron et al. 2012, Thaiyalnayaki y Sowmeyan 2012).

Dentro de los sistemas de tratamiento de aguas residuales mediante un lecho fijo se encuentran los reactores de lecho fluidizado inverso (LFI), mismos que se han sugerido para el tratamiento anaerobio de efluentes industriales (Alvarado et al. 2010, Abdurahman et al. 2013). El LFI utiliza como soporte pequeñas partículas con una densidad específica menor que la del agua, estas partículas flotan y son expandidas hacia abajo por el influente (Sowmeyan y Swaminathan 2008). En el caso de la producción de gas, éste también contribuye a la expansión del lecho, fenómeno llamado pseudofluidización (Arnaiz et al. 2005). La fluidización inversa tiene ventajas adicionales comparadas con la fluidización convencional, entre ellas, permite la recuperación de sólidos en el fondo 
del reactor. En este sentido, la biopelícula que crece en la parte superior del reactor permanece separada de los precipitados, así el reactor no está propenso a atascamientos y tiene menor requerimiento de energía (Papirio et al. 2012). Por otro lado, se considera que los reactores LFI pueden alcanzar una carga orgánica volumétrica superior a $30 \mathrm{~g} \mathrm{DQO} / \mathrm{L}$ d (Alvarado et al. 2008). Este proceso ha sido probado a nivel laboratorio con éxito sobre aguas residuales urbanas, aguas residuales de la industria vinícola (Sowmeyan y Swaminathan 2008, Choudhury y Sahoo 2012, Thaiyalnayaki y Sowmeyan 2012) y aguas residuales de cervecería (Alvarado et al. 2008), así como a escala real en aguas residuales de la industria refresquera, textil y de productos químicos (Yougsheng 2008). El presente trabajo describe la operación de un reactor de lecho fluidizado inverso, empleando Extendospher ${ }^{\circledR}$ como soporte y tratando efluentes provenientes de la producción de etanol a partir de melaza de azúcar de caña. El desempeño del reactor se evaluó aplicando diferentes cargas orgánicas volumétricas y diferentes tiempos de residencia hidráulica.

\section{MATERIALES Y MÉTODOS}

\section{Caracterización del agua residual en estudio}

Durante el desarrollo de este trabajo se llevaron a cabo muestreos simples del efluente proveniente de una destilería productora de etanol a partir de melaza de azúcar de caña. La empresa alcoholera generadora de las vinazas está ubicada en el centro del estado de Veracruz, México y tiene una capacidad de producción de alcohol de $20000 \mathrm{~L} / \mathrm{d}$. Los muestreos se llevaron a cabo directamente en el punto de des- carga hacia una laguna, utilizada como laguna de enfriamiento y homogeneización del efluente. La caracterización de las vinazas provenientes de la producción de etanol, empleadas como influente del LFI, se realizó de acuerdo con técnicas analíticas de los métodos estandarizados para el análisis de aguas y aguas residuales (APHA 1995). Se evaluaron los siguientes parámetros: demanda bioquímica de oxígeno $\left(\mathrm{DBO}_{5}\right)$ (Método 5210-B, a través de la medición de oxígeno disuelto con un oxímetro), DQO total y soluble (Método 5220-C, reflujo cerrado espectrofotométrico), sólidos totales (ST), sólidos totales volátiles (STV), sólidos suspendidos totales (SST), sólidos suspendidos volátiles (SSV) (Método 2540, gravimétrico, sólidos en todas sus formas), $\mathrm{pH}$ (Método 4500, electrométrico), conductividad (Método 2510-B, determinación de la conductividad electrolítica), nitrógeno total Kjeldahl (NTK), nitrógeno amoniacal $\left(\mathrm{N}^{-\mathrm{NH}_{4}}\right.$ ) y nitrógeno orgánico (Método 4500-B, determinación de NTK), fosfatos (Método 4500-P-D, determinación de fósforo total, método de cloruro estañoso) y sulfatos (Método 4500- $\mathrm{SO}_{4}{ }^{2-} \mathrm{E}$, espectrofotométrico de cloruro de bario).

\section{Arranque del reactor de lecho fluidizado inverso}

El dispositivo experimental, LFI, estuvo conformado por una columna de vidrio con una altura de $0.55 \mathrm{~m}$, un diámetro interno de $0.07 \mathrm{~m}$ y un volumen útil de 1.7 L (Fig. 1a y b). Como soporte se empleó Extendosphere ${ }^{\circledR}$, que consiste en un material mineral de forma granular, compuesto principalmente por sílice. El diámetro promedio de estas esferas de soporte es de 170 $\mu \mathrm{m}$, su densidad es igual a $690 \mathrm{~kg} / \mathrm{m}^{3}$, con un área superficial específica igual a $20000 \mathrm{~m}^{2} / \mathrm{m}^{3}$

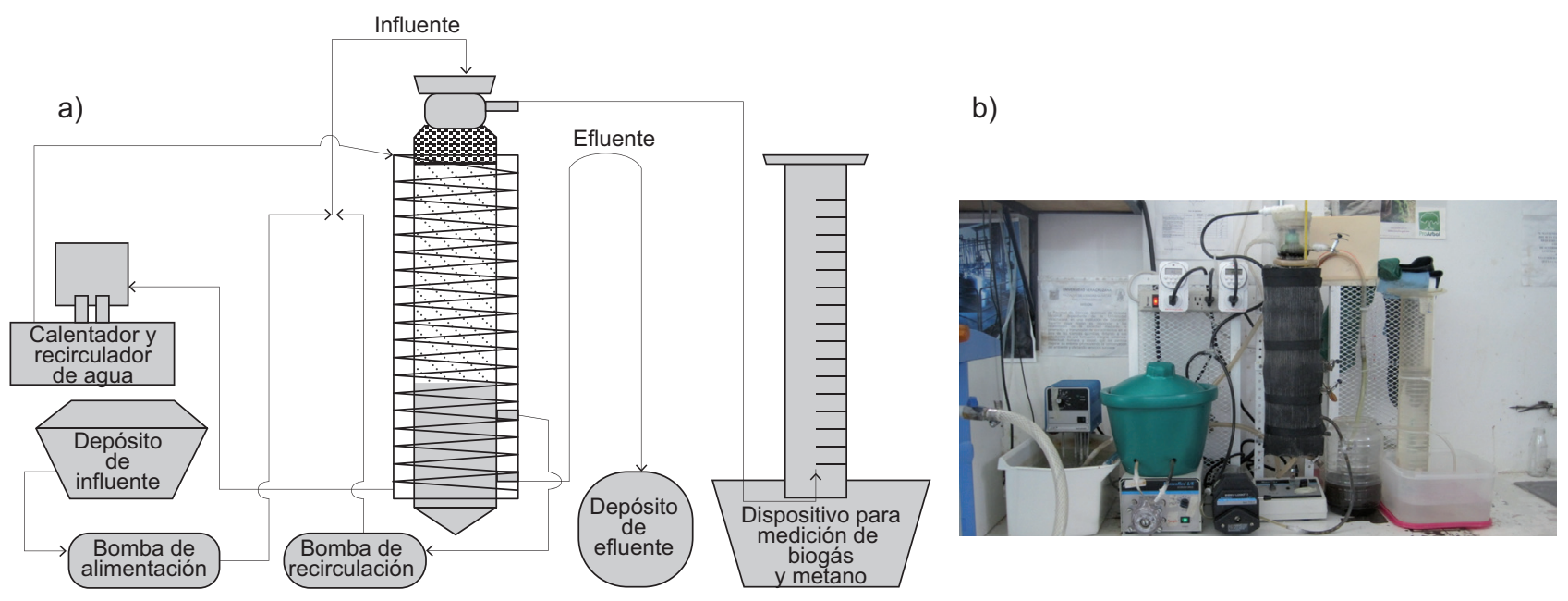

Fig. 1. Reactor de lecho fluidizado inverso anaerobio (LFI) empleado como prototipo experimental 
CUADRO I. CONDICIONES DE OPERACIÓN POR LOTES PARA EL LECHO FLUIDIZADO INVERSO ANAEROBIO (LFI)

\begin{tabular}{lclc}
\hline Variable de operación & Valor & Variable de operación & Valor \\
\hline Concentración de entrada & $0.5 \mathrm{~g} \mathrm{DQO} / \mathrm{L}$ & Volumen de soporte seco & $0.247 \mathrm{~L}$ \\
Área de la columna & $0.0038 \mathrm{~m}^{2}$ & Porcentaje de soporte seco & $14.5 \%$ \\
Velocidad descencional & $7.34 \mathrm{~m} / \mathrm{h}$ & Altura del líquido & $0.46 \mathrm{~m}$ \\
Flujo de recirculación & $28.2 \mathrm{~L} / \mathrm{h}$ & Relación Ho/D & 6.57 \\
Volumen útil del reactor & $1.7 \mathrm{~L}$ & Temperatura de operación & $30^{\circ} \mathrm{C}$. \\
\hline
\end{tabular}

$\mathrm{DQO}=$ Demanda química de oxígeno, $\mathrm{Ho} / \mathrm{D}=$ Relación altura/diámetro

(Arnaiz et al. 2007). Se emplearon $0.247 \mathrm{~L}$ de soporte seco, que corresponden a un área superficial para adhesión de biopelícula de $4.94 \mathrm{~m}^{2}$. El reactor se adaptó con un dispositivo distribuidor del líquido de alimentación en la parte superior de la columna. En la parte inferior derecha del reactor se ubicó la salida del efluente. Para la alimentación y la recirculación se emplearon bombas peristálticas de velocidad variable. El biogás producido se midió con un sistema de desplazamiento de volumen de líquido tipo frasco de Mariotte. Se utilizó el mismo procedimiento para la cuantificación del metano producido con $\mathrm{NaOH}$ para el lavado del biogás.

El reactor se inoculó con $0.250 \mathrm{~L}$ de lodos húmedos (sedimentados y lavados), con una concentración de $48.44 \mathrm{~g} / \mathrm{L}$ de SSV. Los lodos fueron obtenidos de una planta de tratamiento de aguas residuales que opera bajo un sistema anaerobio.

Después de la inoculación del reactor se inició su operación por lotes, con un influente de una concentración igual a $0.5 \mathrm{~g} \mathrm{DQO} / \mathrm{L}$. Se mantuvo en esta condición durante 51 días, periodo en el que se completaron cinco ciclos de 13, 7, 11, 10 y 10 días, hasta alcanzar una eficiencia de remoción casi constante. Se realizó un monitoreo de la DQO soluble del influente y el efluente. Las condiciones de operación en lotes se muestran en el cuadro I. Durante la operación en lotes se dio seguimiento a la colonización del soporte, con observaciones directas al microscopio. Para este fin, las muestras fueron tomadas de los diversos puertos de muestreo ubicados a diferentes alturas en el reactor.

\section{Operación a diferente carga orgánica volumétrica}

Una vez que se obtuvo un soporte colonizado, se operó el reactor en continuo alimentando el agua residual en estudio sin diluir (cruda, excepto para las cargas de $0.5 \mathrm{~g} \mathrm{DQO} / \mathrm{L}$ d y $1.0 \mathrm{~g} \mathrm{DQO} / \mathrm{L}$ d). Se efectuaron incrementos de la carga orgánica volumétrica desde $0.5 \mathrm{~g} \mathrm{DQO} / \mathrm{L}$ d hasta $10.4 \mathrm{~g}$ $\mathrm{DQO} / \mathrm{L}$ d, cuando se alcanzó la estabilización del sistema para cada carga evaluada. Los valores del tiempo de residencia hidráulica fueron variables, tal como se muestra en el cuadro II, en donde se indican también los valores del flujo del influente y de recirculación empleados en cada carga orgánica volumétrica. Durante la operación en continuo se monitorearon los siguientes parámetros: $\mathrm{DQO}, \mathrm{ST}$, STV, SST, pH, volumen de biogás y volumen de metano. El crecimiento de la biopelícula también fue monitoreado con observaciones al microscopio, cuando se alcanzó la estabilidad en el sistema. Asimismo, se determinaron los sólidos volátiles adheridos al soporte (SVA).

CUADRO II. OPERACIÓN DEL LECHO FLUIDIZADO INVERSO ANAEROBIO (LFI) EN CONTINUO A DIFERENTES CARGAS ORGÁNICAS VOLUMÉTRICAS

\begin{tabular}{|c|c|c|c|c|c|c|}
\hline \multirow{2}{*}{$\begin{array}{l}\text { Carga orgánica } \\
\text { volumétrica } \\
\text { g DQO/L.d }\end{array}$} & \multirow{2}{*}{$\begin{array}{c}\text { Carga orgánica } \\
\text { específica } \\
\text { gDQO } / \mathrm{m}^{2} \cdot \mathrm{d}\end{array}$} & \multicolumn{2}{|c|}{$\begin{array}{c}\mathrm{Q} \\
\mathrm{L} / \mathrm{d}\end{array}$} & \multirow{2}{*}{$\begin{array}{l}\text { Valores de } \\
\text { recirculación } \\
\mathrm{L} / \mathrm{h}\end{array}$} & \multirow{2}{*}{$\begin{array}{c}\mathrm{TRH} \\
\text { (días) }\end{array}$} & \multirow{2}{*}{$\begin{array}{c}\text { Tiempo de } \\
\text { operación } \\
\text { (días) }\end{array}$} \\
\hline & & Promedio & $\begin{array}{l}\text { Desviación } \\
\text { estándar }\end{array}$ & & & \\
\hline 0.5 & 0.1012 & 0.922 & 0.0 & 28.2 & 1.8 & 31 \\
\hline 1.0 & 0.2024 & 0.039 & 0.006 & 28.2 & 50 & 105 \\
\hline 3.3 & 0.6680 & 0.052 & 0.012 & 24.0 & 34 & 119 \\
\hline 6.8 & 1.377 & 0.073 & 0.008 & 19.8 & 23 & 45 \\
\hline 10.4 & 2.105 & 0.081 & 0.0 & 15.0 & 21 & 14 \\
\hline
\end{tabular}

Q = Flujo de alimentación, TRH: Tiempo de residencia hidráulica, DQO: Demanda química de oxígeno 
CUADRO III. CARACTERÍSTICAS DE LAS VINAZAS GENERADAS EN LA PRODUCCIÓN DE ETANOL

\begin{tabular}{lcclcc}
\hline \multirow{2}{*}{ Parámetro } & \multicolumn{2}{c}{ Valor } & \multicolumn{2}{c}{ Valor } \\
\cline { 2 - 4 } & Promedio & Desviación estándar & & Promedio & Desviación estándar \\
\cline { 2 - 5 } Temperatura $\left({ }^{\circ} \mathrm{C}\right)$ & 96 & 3.95 & SST $(\mathrm{g} / \mathrm{L})$ & 14.71 & 2.69 \\
$\mathrm{pH}$ & 4.64 & 0.28 & SSV $(\mathrm{g} / \mathrm{L})$ & 10.90 & 1.86 \\
Conductividad $(\mathrm{mS} / \mathrm{cm})$ & 19.48 & 8.66 & NTK $(\mathrm{g} / \mathrm{L})$ & 0.259 & 0.052 \\
DQO $_{\mathrm{t}}(\mathrm{g} / \mathrm{L})$ & 193.35 & 54.64 & Nitrógeno Orgánico $\mathrm{g} / \mathrm{L}$ & 0.177 & 0.033 \\
DQO $_{\mathrm{s}}(\mathrm{g} / \mathrm{L})$ & 179.37 & 42.06 & Nitrógeno amoniacal g/L & 0.082 & 0.021 \\
$\mathrm{ST}(\mathrm{g} / \mathrm{L})$ & 109.78 & 34.41 & Fósforo $\mathrm{g} / \mathrm{L}$ & 0.218 & 0.010 \\
$\mathrm{STV}(\mathrm{g} / \mathrm{L})$ & 77.53 & 25.93 & Sulfatos $\mathrm{g} / \mathrm{L}$ & 11.49 & 3.89 \\
\hline
\end{tabular}

$\mathrm{SST}=$ Sólidos suspendidos totales, $\mathrm{SSV}=$ Sólidos suspendidos volátiles, NTK $=$ Nitrógeno total Kjedahl, $\mathrm{DQO}_{\mathrm{t}}=\mathrm{Demanda}$ química de oxígeno total, $\mathrm{DQO}_{\mathrm{s}}=$ Demanda química de oxígeno solubre, $\mathrm{ST}=$ Sólidos totales, $\mathrm{STV}=$ Sólidos totales volátiles

\section{Operación a diferentes tiempos de residencia hidráulica (TRH)}

Cuando el reactor se estabilizó a la máxima carga orgánica volumétrica bajo la cual operó eficientemente, se disminuyó gradualmente el TRH. Para lo anterior, se operó el reactor con una carga de $6.4 \mathrm{~g}$ $\mathrm{DQO} / \mathrm{L}$ d, a diferentes TRH de 10, 5 y 1 días, cada uno con periodos de 30, 20 y 10 días respectivamente. Para mantener constante el TRH se controló el flujo de alimentación y mediante diluciones se ajustó la concentración de DQO de la vinaza empleada como influente.

\section{RESULTADOS Y DISCUSIÓN}

De los muestreos y caracterizaciones realizados cada tres meses en la planta productora de etanol (un total de nueve muestreos), se obtuvieron los valores promedio para los diversos parámetros evaluados que se muestran en el cuadro III. El efluente estaba compuesto en su mayoría por vinazas, además, contenía una menor proporción de agua proveniente del lavado de los diferentes tanques del proceso (tales como los de preparación de mosto, de producción de levadura y de fermentación). Se observa un valor bajo de $\mathrm{pH}$ en el efluente caracterizado, causado por las cantidades de ácido sulfúrico empleado en el proceso (específicamente en la preparación del mosto) para evitar la presencia de bacterias durante la producción de levadura. Por otro lado, se observan valores elevados de DQO, mismos que rebasan los reportados por diversos autores y que se encuentran en rangos de 50 a 122 g/L DQO (Vlyssides y Barampounti 2005, Jiménez et al. 2006, Travieso et al. 2006). En relación con la cantidad de sólidos en el efluente crudo, éstos se encuentran dentro de los rangos reportados, los cuales presentan valores entre 70.6 a $170 \mathrm{~g} / \mathrm{L}$ para los ST y 3.6 a $145 \mathrm{~g} / \mathrm{L}$ para los SST (Vlyssides y Barampounti et al. 2005, Jiménez et al. 2006, Travieso et al. 2006). Respecto a los valores de nitrógeno, son más bajos que los reportados por algunos autores, los cuales están entre 1.8 a $170 \mathrm{~g} / \mathrm{L}$ para NTK(Vlyssides y Barampounti 2005, Jiménez et al. 2006) y $48 \mathrm{~g} / \mathrm{L}$ para $\mathrm{N}_{-} \mathrm{NH}_{4}$ (Vlyssides y Barampounti 2005). Al igual que en el caso de la DQO, una gran cantidad de sólidos no tienen su origen únicamente en el efluente de la destilación (estrictamente vinazas), ya que provienen también de sólidos sedimentables de la melaza empleada como sustrato y que no son utilizados durante la fermentación, así como de los efluentes provenientes del lavado de fermentadores, que contienen una gran cantidad de levadura que no es centrifugada.

La operación en lotes del LFI se llevó a cabo durante 51 días, en cinco ciclos. La duración de cada uno estuvo en función de la concentración del efluente del reactor. La mayor eficiencia de remoción de DQO alcanzada fue de $88 \%$, conseguida tanto en el segundo, como en el último ciclo. Se consideró que el sistema alcanzó estabilidad operando en lotes cuando los porcentajes de remoción de DQO fueron superiores a $80 \%$ en los últimos dos ciclos. (Cuadro IV). Durante la operación del LFI en sistema lotes no hubo generación de metano.

Respecto a la colonización del soporte, las observaciones realizadas al microscopio demostraron un incremento de biomasa adherida al Extendosphere ${ }^{\circledR}$ durante la operación del reactor en lotes. En la figura 2a se presenta una observación al microscopio (con un aumento $10 \mathrm{x}$ ) del soporte sin colonizar al inicio de la operación del reactor en lotes, así como el soporte colonizado a diferentes tiempos de operación en las figuras $\mathbf{2 b}, \mathbf{c}$ y $\mathbf{d}$. Durante el primer ciclo se observa apenas el inicio de la colonización (a los 8 días), en el que ocurrió un aumento considerable de la biomasa 
CUADRO IV. EFICIENCIAS DE REMOCIÓN ALCANZADAS EN EL REACTOR DE LECHO FLUIDIZADO INVERSO ANAEROBIO OPERANDO POR LOTES

\begin{tabular}{cccccc}
\hline $\begin{array}{c}\text { Número } \\
\text { de lote }\end{array}$ & $\begin{array}{c}\text { Tiempo acumulado } \\
\text { (días) }\end{array}$ & $\begin{array}{c}\text { DQO influente } \\
\mathrm{g} / \mathrm{L}\end{array}$ & $\begin{array}{c}\text { DQO efluente } \\
\mathrm{g} / \mathrm{L}\end{array}$ & $\begin{array}{c}\text { DQO removida } \\
\mathrm{g} / \mathrm{L}\end{array}$ & $\begin{array}{c}\text { Porcentaje de } \\
\text { remoción }(\%)\end{array}$ \\
\hline 1 & 13 & 0.5952 & 0.215 & 380.11 & 63.87 \\
2 & 20 & 0.5334 & 0.062 & 471.03 & 88.31 \\
3 & 31 & 0.5204 & 0.106 & 413.84 & 79.52 \\
4 & 41 & 0.5204 & 0.066 & 454.00 & 87.23 \\
5 & 51 & 0.5567 & 0.065 & 491.50 & 88.29 \\
\hline
\end{tabular}

$\mathrm{DQO}=$ Demanda química de oxígeno

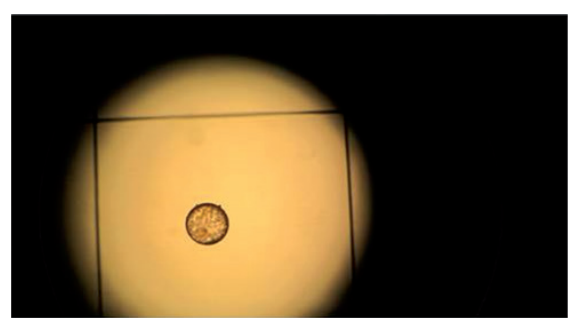

(a) Soporte sin colonizar al inicio de la operación en lotes

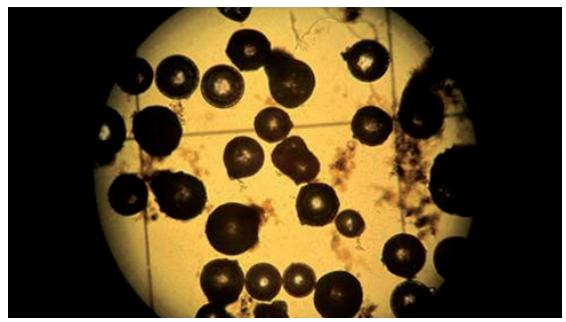

(c) Crecimiento de biopelícula durante el tercer lote

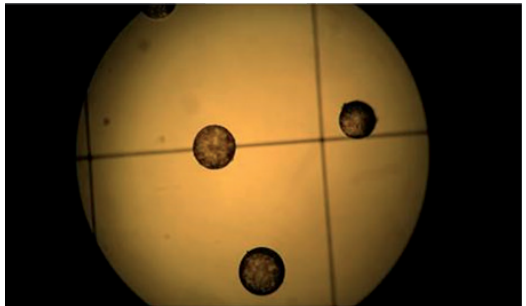

(b) Soporte con biomasa adherida a los 8 días de operación en lotes

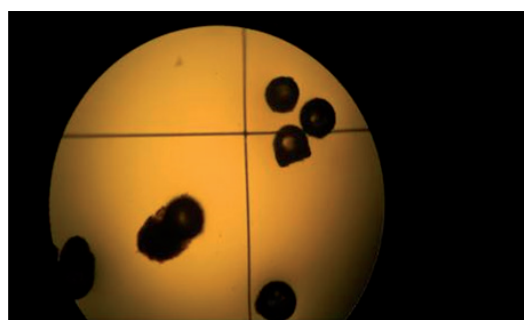

(d) Biopelícula adherida al soporte durante el cuarto lote

Fig. 2. Soporte (Extendosphere ${ }^{\circledR}$ ) visto al microscopio con aumento 10 x. (a) Soporte sin colonizar al arranque del reactor, (b) Soporte durante el primer ciclo por lote, 8 días de operación, (c) Colonización del soporte a los 25 días de operación en lote, (d) Biomasa adherida al soporte al final del cuarto lote, 40 días

adherida durante el tercer ciclo, lo que coincidió con un incremento en la eficiencia de remoción de DQO. Thaiyalnayaki y Sowmeyan (2012), han considerado que el desarrollo de la biomasa y su adherencia al soporte son factores importantes que determinan el eficiente desempeño de un reactor de lecho fluidizado inverso anaerobio. De acuerdo con Michaud et al. (2005) y Houbron et al. (2012), el periodo de arranque de un reactor puede ser visto como el tiempo necesario para la selección y justo arreglo de la más apropiada variedad de bacterias de un consorcio, en donde están relacionadas unas a otras dependiendo del sustrato. Durante el periodo de arranque de un reactor de lecho fijo, el proceso de colonización se inicia con la adhesión de algunas células de bacterias a la superficie del soporte en una fase de adhesión, seguida por una fase de colonización, en donde se presenta una comunicación intercelular, crecimiento y formación de polisacáridos. En una siguiente fase el crecimiento celular aumenta, así como la cantidad de polisacáridos que permite la adhesión, creando una biopelícula de mayor espesor y más resistente al desprendimiento de las células que crecen sobre las superficies expuestas a corrientes de líquidos.

Las condiciones bajo las cuales operó el reactor a diferentes cargas y las eficiencias alcanzadas en cada condición, se muestran en el cuadro $\mathbf{V}$. La operación en continuo se inició con una carga de $0.5 \mathrm{~g}$ $\mathrm{DQO} / \mathrm{Ld}$. El TRH fue de 1.8 días y la concentración de vinaza alimentada fue ajustada a $0.9 \mathrm{~g} \mathrm{DQO} / \mathrm{L}$ (o valores muy cercanos a éste), con diluciones de vinaza. Asimismo, se controló el flujo del influente. La eficiencia de remoción de DQO promedio fue de $58 \%$, con un valor máximo de $84 \%$. Durante la 
CUADRO V. EFICIENCIAS DE REMOCIÓN ALCANZADAS EN EL REACTOR DE LECHO FLUIDIZADO INVERSO ANAEROBIO (LFI) A DIFERENTES CARGAS ORGÁNICAS VOLUMÉTRICAS

\begin{tabular}{|c|c|c|c|c|c|c|c|c|c|c|c|}
\hline \multicolumn{2}{|c|}{$\begin{array}{c}\text { DQO } \\
\text { influente } \\
(\mathrm{g} \mathrm{DQO} / \mathrm{L})\end{array}$} & \multicolumn{2}{|c|}{$\begin{array}{c}\text { DQO } \\
\text { efluente } \\
(\mathrm{g} \text { DQO/L) }\end{array}$} & \multicolumn{2}{|c|}{$\begin{array}{c}\text { DQO } \\
\text { removida } \\
(\mathrm{g} \mathrm{DQO} / \mathrm{L})\end{array}$} & \multicolumn{2}{|c|}{$\begin{array}{l}\text { Carga orgánica } \\
\text { volumética } \\
\text { (g DQO/L d) }\end{array}$} & \multicolumn{2}{|c|}{$\begin{array}{l}\text { Eficiencia de } \\
\text { remoción } \\
(\%)\end{array}$} & \multirow{2}{*}{$\begin{array}{l}\text { T. O. } \\
\text { L.F.I. } \\
\text { (días) }\end{array}$} & \multirow[t]{2}{*}{$\begin{array}{r}\text { TRH } \\
\text { (días) }\end{array}$} \\
\hline Promedio & D. E. & Promedio & D. E. & Promedio & D. E. & Promedio & D. E. & Promedio & D. E. & & \\
\hline 0.91 & 0.14 & 0.35 & 0.12 & 0.38 & 0.22 & 0.50 & 0.08 & 58.00 & 20.78 & 45 & 1.80 \\
\hline 50.63 & 14.78 & 12.37 & 5.25 & 16.510 & 10.67 & 1.00 & 0.10 & 67.00 & 12.19 & 105 & 50.00 \\
\hline 109.27 & 23.47 & 43.71 & 8.46 & 64.404 & 27.10 & 3.30 & 0.13 & 59.00 & 11.85 & 119 & 34.00 \\
\hline 163.69 & 28.96 & 84.78 & 24.18 & 80.970 & 22.98 & 6.80 & 0.27 & 50.00 & 7.56 & 45 & 23.00 \\
\hline 218.51 & 0.0 & 153.03 & 15.29 & 65.48 & 15.29 & 10.4 & 0.0 & 29.97 & 21.22 & 14 & 21.00 \\
\hline
\end{tabular}

$\mathrm{DQO}=$ Demanda química de oxígeno, TRH $=$ Tiempo de residencia hidráulica, D. E. $=$ Desviación estándar, T. O. = Tiempo de operación del reactor de lecho fluidizado inverso

operación con esta carga orgánica volumétrica no hubo producción de biogás. Cuando se alcanzó una remoción de DQO casi constante, se incrementó la carga. La concentración del influente para la carga de $1.0 \mathrm{~g} \mathrm{DQO} / \mathrm{L}$ d fue ajustada con diluciones de la vinaza a $50 \mathrm{~g} \mathrm{DQO} / \mathrm{L}$, manteniendo un control del flujo de entrada, con lo que se consiguió un TRH de 50 días. El caudal de alimentación y la tasa de recirculación se indican en el cuadro II. En la figura 3 se observa que al operar el reactor a la carga de $1.0 \mathrm{~g}$ DQO/L d, durante los días 87 a 127, la eficiencia de remoción se mantuvo muy cercana al $80 \%$ o por arriba de este valor. Sin embargo, entre el día 128 y 141, la eficiencia disminuyó hasta un valor aproximado a $60 \%$. A partir del día 143 y hasta el final de la operación a esta carga, el sistema parece alcanzar estabilidad, pues la variación en la eficiencia de remoción disminuyó. Finalmente, la eficiencia promedio de remoción a esta carga fue de $67 \%$ y se cumplieron dos TRH. Durante la operación del reactor a una carga de $3.3 \mathrm{~g} \mathrm{DQO} / \mathrm{L}$ d, se utilizó como influente vinaza cruda sin diluir, obtenida durante

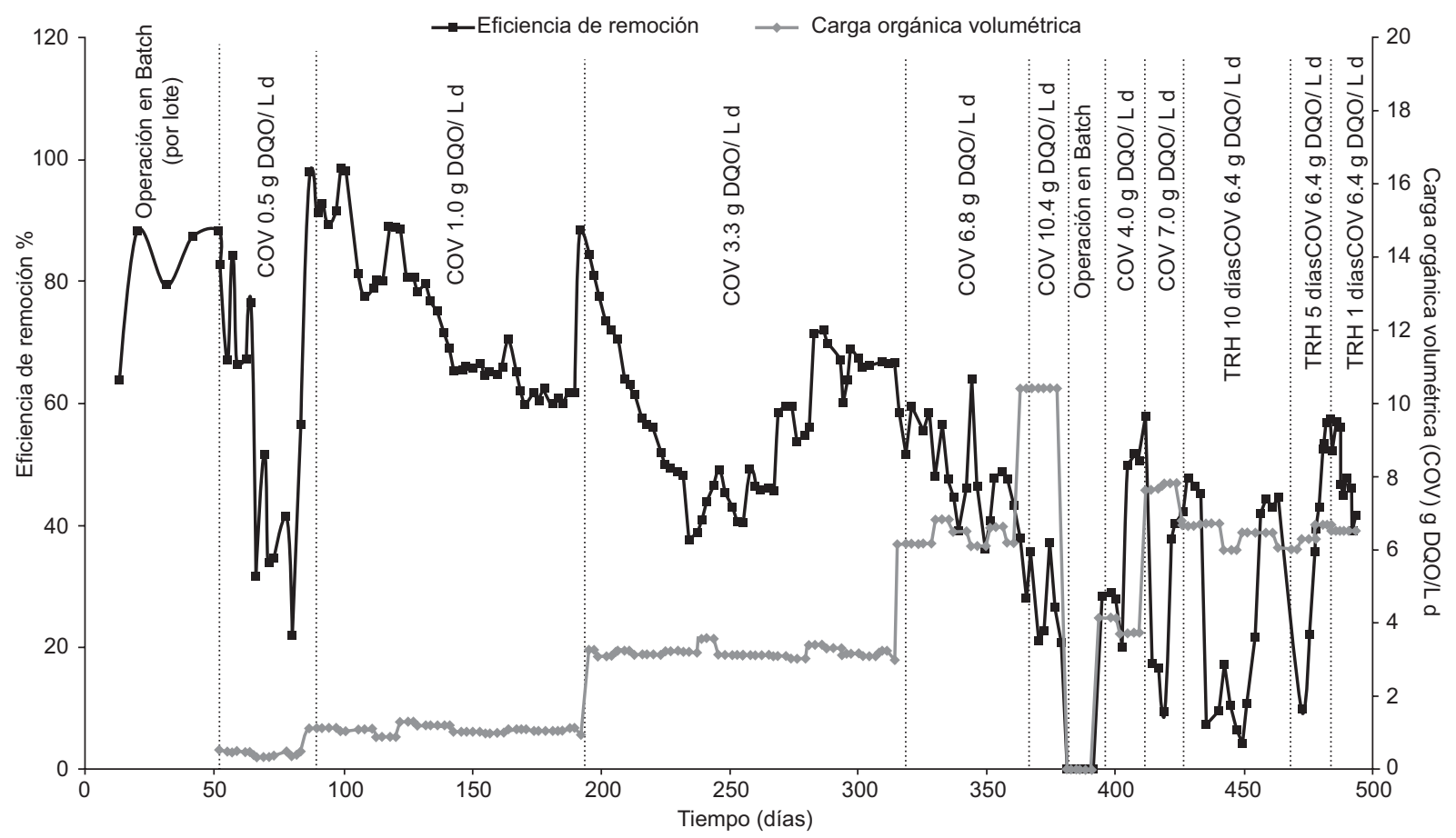

Fig. 3. Eficiencias de remoción obtenidas en el reactor de lecho fluidizado inverso anaerobio (LFI) a diferentes cargas orgánicas volumétricas. $\mathrm{COV}=$ Carga orgánica volumétrica, $\mathrm{TRH}=$ Tiempo de residencia hidráulica, $\mathrm{DQO}=$ Demanda química de oxígeno 
los muestreos en la destilería. En la caracterización de esta vinaza se encontraron diferentes valores de DQO, en un rango de $73.0 \mathrm{~g}$ DQO/L hasta $130.0 \mathrm{~g}$ DQO/L. De tal manera que, para lograr la carga deseada en el LFI, que mantenía un volumen fijo de $1.7 \mathrm{~L}$, fue necesario calcular el flujo de alimentación para las diferentes concentraciones de DQO de la vinaza empleada como influente. En la figura 3 puede apreciarse que la operación del reactor a esta carga permitió alcanzar una estabilidad después de operar 119 días, así como una eficiencia de remoción de DQO promedio de $59 \%$. El tiempo de residencia fue de 34 días y se cumplieron tres TRH.

Con la finalidad de probar la tolerancia del LFI a una mayor carga orgánica volumétrica, se operó el reactor a $6.8 \mathrm{~g} \mathrm{DQO} / \mathrm{L} \mathrm{d}$ con vinaza cruda (DQO promedio de $163.7 \mathrm{~g} \mathrm{DQO} / \mathrm{L}$ ). Dado que se mantenía un volumen fijo de $1.7 \mathrm{~L}$ en el reactor, fue necesario determinar el valor de flujo del influente. Derivado de este flujo, el TRH fue de 20 días y debido a que el reactor se operó bajo estas condiciones durante 45 días, se cumplieron dos tiempos de residencia. Se alcanzó estabilidad a los 29 días de operación. La eficiencia de remoción de DQO promedio fue de $50 \%$. A pesar de que la eficiencia de remoción y el rendimiento de metano disminuyeron, se alcanzó una estabilidad en un corto tiempo. Al operar el reactor a una carga orgánica volumétrica de $10.4 \mathrm{~g} \mathrm{DQO} / \mathrm{L} \mathrm{d}$, se presentó inestabilidad en su operación, lo que causó una disminución en la eficiencia de remoción de DQO hasta un $30 \%$. Asimismo, el pH alcanzó un valor de 5 y fue necesario ajustarlo diariamente (Fig. 3). Se asume que la disminución de $\mathrm{pH}$ puede ser ocasionada por la acumulación de algunos ácidos orgánicos intermediarios que se producen durante el tratamiento anaerobio, tales como los ácidos butírico, propiónico y acético. Debido a los problemas presentados, se suspendió la operación en continuo y se operó el reactor en lotes durante un periodo de 12 días hasta que el $\mathrm{pH}$ del efluente alcanzó un valor cercano a 7.0 y se obtuvo una eficiencia de remoción de DQO promedio de $28 \%$. Después de operar el reactor en lotes, se reinició la operación en continuo a una carga de $4.0 \mathrm{~g} \mathrm{DQO} / \mathrm{L} \mathrm{d}$, alcanzando estabilidad en 16 días, con una eficiencia de remoción promedio de $50 \%$. Posteriormente se incrementó la carga a $7.0 \mathrm{~g}$ $\mathrm{DQO} / \mathrm{L}$ d y se alcanzó una concentración de DQO en el efluente casi constante a los 11 días de operación, con una eficiencia de remoción promedio de $50 \%$ (Fig. 3). Investigaciones anteriores han reportado diferentes eficiencias de remoción de DQO en LFI. Thaiyalnayaki y Sowmeyan (2012), alcanzaron una eficiencia de remoción de DQO de $55 \%$ al operar un reactor de lecho fluidizado inverso anaerobio a una capacidad máxima de $15 \mathrm{~g}$ de $\mathrm{DQO} / \mathrm{L} \mathrm{d}$, tratando aguas residuales de alta carga. Por otro lado, Alvarado et al. (2008), obtuvieron eficiencias de remoción superiores a $80 \%$ con cargas volumétricas de hasta $30 \mathrm{~g} \mathrm{DQO} / \mathrm{Ld}$ en un LFI tratando aguas residuales de la industria cervecera. En el presente trabajo, el valor de DQO del agua residual cruda en estudio empleada como influente en la carga orgánica volumétrica de $10.4 \mathrm{~g} \mathrm{DQO} / \mathrm{L} \mathrm{d}$ fue en promedio, $218.51 \mathrm{~g} \mathrm{DQO} / \mathrm{L}$, la más alta respecto a los valores de concentración de DQO de la vinaza empleada en las diferentes cargas evaluadas. Es importante considerar que las vinazas provenientes de la producción de etanol contienen compuestos recalcitrantes tales como compuestos orgánicos poliaromáticos, polifenólicos, productos de lignina, taninos y ácidos húmicos (Zayas et al. 2010), mismos que impiden una considerable disminución de la DQO al no ser removidos. La presencia de sustancias inhibitorias en las vinazas, tales como los compuestos fenólicos, dificulta el proceso de digestión anaerobia. Es conocido que estos compuestos son tóxicos e interfieren en la actividad de los microorganismos metanogénicos. Además, la alta salinidad de estos efluentes, observada en sus elevados valores de conductividad (en algunos casos de hasta $40 \mathrm{mS} / \mathrm{cm}$ ), pueden causar problemas de presión osmótica a los microorganismos responsables de la digestión anaerobia (Jiménez et al. 2006). Las anteriores consideraciones podrían ser la causa de la desestabilización del sistema al operarlo con la carga de $10.4 \mathrm{~g} \mathrm{DQO} / \mathrm{L} \mathrm{d}$.

La determinación de la cantidad de SVA al soporte por gramo de soporte ( $\left.\mathrm{g}_{\mathrm{SVA}} / \mathrm{g}_{\mathrm{SOPORTE}}\right)$, se realizó a partir de la operación del reactor en continuo a una carga de $3.3 \mathrm{~g} \mathrm{DQO} / \mathrm{L} \mathrm{d}$ (y para todas las cargas posteriores), considerando que se había conseguido estabilidad en la operación del reactor en esta carga y que habría suficiente biomasa colonizando el soporte para poder ser cuantificada por este método. La cantidad de SVA se mantuvo en un rango desde 0.14 $\mathrm{g}_{\mathrm{SVA}} / \mathrm{g}_{\mathrm{SOPORTE}}$ hasta $0.26 \mathrm{~g}_{\mathrm{SVA}} / \mathrm{g}_{\text {SOPORTE }}$. La menor cantidad de SVA coincide con el periodo de desestabilización del reactor a una carga de $10.4 \mathrm{~g} \mathrm{DQO} / \mathrm{Ld}$, en donde el valor de $\mathrm{pH}$ disminuyó hasta un valor de 5, así como la producción de metano. La cantidad se incrementó nuevamente al operar el reactor en lotes y en la posterior operación en continuo a $4.0 \mathrm{~g} \mathrm{DQO} / \mathrm{Ld}$ y $7.0 \mathrm{~g} \mathrm{DQO} / \mathrm{L}$ d. Asimismo, se obtuvieron valores cercanos al operar el reactor a una carga orgánica volumétrica promedio de $6.4 \mathrm{~g} \mathrm{DQO} / \mathrm{L}$ d bajo TRH de 10,5 y 1 días. El valor promedio fue de 0.20 $\mathrm{g}_{\mathrm{SVA}} / \mathrm{g}_{\text {SOPORTE}}$. Los valores de biomasa adherida al 
Extendosphere ${ }^{\circledR}$ son semejantes a los reportados por Thaiyalnayaki y Sowmeyan (2012) al emplear un rector de lecho fluidizado inverso, utilizando como soporte esferas de vidrio para tratar aguas residuales de alta carga.

Cuando el reactor recuperó su estabilidad y con la finalidad de optimizar su desempeño, se operó a diferentes TRH, los cuales se fueron disminuyendo gradualmente bajo una carga promedio de $6.4 \mathrm{~g}$ $\mathrm{DQO} / \mathrm{L}$ d, ya que fue dicha carga bajo la que operó eficientemente. Con un TRH de 10 días, se alcanzó una eficiencia de remoción promedio igual a $45 \%$ al final del periodo. Bajo esta condición, fue necesario ajustar el $\mathrm{pH}$ diariamente.

Cuando el reactor operó durante un periodo de tiempo de 20 días y a un TRH de cinco días, se completaron cuatro tiempos de residencia. Durante los primeros tres TRH evaluados se presentaron menos cambios en el $\mathrm{pH}$. En el último TRH evaluado no fue necesario ajustar el $\mathrm{pH}$. Adicionalmente, se obtuvo una eficiencia de remoción de DQO promedio de $57.42 \%$. Cuando el reactor se operó a un TRH igual a un día, se obtuvo una eficiencia de remoción de DQO promedio de $52 \%$. Sin embargo, se observa una tendencia a seguir disminuyendo hasta alcanzar una eficiencia de $40 \%$, completándose diez tiempos de residencia. Se observó estabilidad en la concentración del efluente y del $\mathrm{pH}$. Así como inestabilidad en el sistema cada vez que se cambió el TRH, ya que la eficiencia de remoción bajó considerablemente, pero se recuperó al final de cada periodo de operación a diferentes TRH (Fig. 3) alcanzando eficiencias de remoción menos variables.

Los valores del rendimiento de metano y su comportamiento durante la operación del reactor a diferentes cargas orgánicas volumétricas se muestran en el cuadro VI y figura 4 respectivamente. Puede observarse que durante la operación del LFI en lotes y en continuo a una carga de $0.5 \mathrm{~g} \mathrm{DQO} / \mathrm{Ld}$, no hubo generación de metano, iniciándose la producción del mismo hasta los 110 días de operación del sistema a una carga de $1.0 \mathrm{~g}$ DQO/L d. Se observa una variación considerable del rendimiento de metano al inicio de su producción, pero se estabiliza al final de la etapa operando a esta carga. En las cargas de 3.3 y 6.8 g $\mathrm{DQO} / \mathrm{L}$ d, la producción de biogás presenta comportamientos similares: se observa claramente que al incrementar las cargas el rendimiento de metano disminuye, pero al final de la operación en estas dos cargas orgánicas volumétricas, la cantidad de metano aumenta manteniendo valores con menos variación. El sistema alcanza valores de rendimiento de metano promedio de 0.32 y $0.24 \mathrm{LCH}_{4} / \mathrm{gDQO}_{\text {removida }}$ para las cargas de 3.3 y $6.8 \mathrm{~g} \mathrm{DQO} / \mathrm{L}$ d, respectivamente. $\mathrm{Al}$ operar el reactor a una carga de $10.4 \mathrm{~g} \mathrm{DQO} / \mathrm{Ld}$, el rendimiento de metano empieza a disminuir hasta desaparecer, mostrando un desequilibrio en el LFI, lo que indica una máxima carga bajo la cual ya no podía ser operado. Una posterior operación del sistema en lotes y con cargas de 4.0 y $7.0 \mathrm{~g} \mathrm{DQO} / \mathrm{L} \mathrm{d}$ permitieron un nuevo incremento en el rendimiento de metano. Después de estos eventos, el reactor

CUADRO VI. RENDIMIENTO DE METANO OBTENIDO A DIFERENTES CARGAS ORGÁNICAS VOLUMÉTRICAS EN EL REACTOR DE LECHO FLUIDIZADO INVERSO (LFI)

\begin{tabular}{|c|c|c|c|c|}
\hline \multirow{2}{*}{$\begin{array}{l}\text { Tiempo de } \\
\text { operación } \\
\text { (Días) }\end{array}$} & \multicolumn{2}{|c|}{$\begin{array}{l}\text { Carga orgánica volumétrica } \\
\text { g DQO/L d }\end{array}$} & \multicolumn{2}{|c|}{$\begin{array}{c}\text { Rendimiento de } \mathrm{CH}_{4}, \mathrm{y}\left(\mathrm{LCH}_{4} / \mathrm{g} \text { DQO removida) }\right. \\
\text { Promedio }\end{array}$} \\
\hline & Promedio & $\begin{array}{l}\text { Desviación } \\
\text { estándar }\end{array}$ & Promedio & $\begin{array}{l}\text { Desviación } \\
\text { estándar }\end{array}$ \\
\hline 51 & Operación por lote & & & $*$ \\
\hline 45 & 0.50 & 0.08 & 0.0 & $*$ \\
\hline 105 & 1.00 & 0.10 & 0.3076 & 0.052 \\
\hline 119 & 3.30 & 0.13 & 0.3156 & 0.046 \\
\hline 45 & 6.80 & 0.27 & 0.2383 & 0.059 \\
\hline 14 & 10.40 & 0.0 & 0.1819 & 0.0 \\
\hline 3 & Operación por lote & & & $*$ \\
\hline 17 & 4.0 & 0.22 & 0.2201 & 0.016 \\
\hline 18 & 7.0 & 0.55 & 0.1736 & 0.050 \\
\hline 30 & 6.4 & 0 & 0.2123 & 0.014 \\
\hline 20 & 6.4 & 0 & 0.1922 & 0.042 \\
\hline 10 & 6.4 & 0 & 0.1488 & 0.021 \\
\hline
\end{tabular}

*No hubo generación de $\mathrm{CH}_{4} ; \mathrm{DQO}=$ Demanda química de oxígeno 


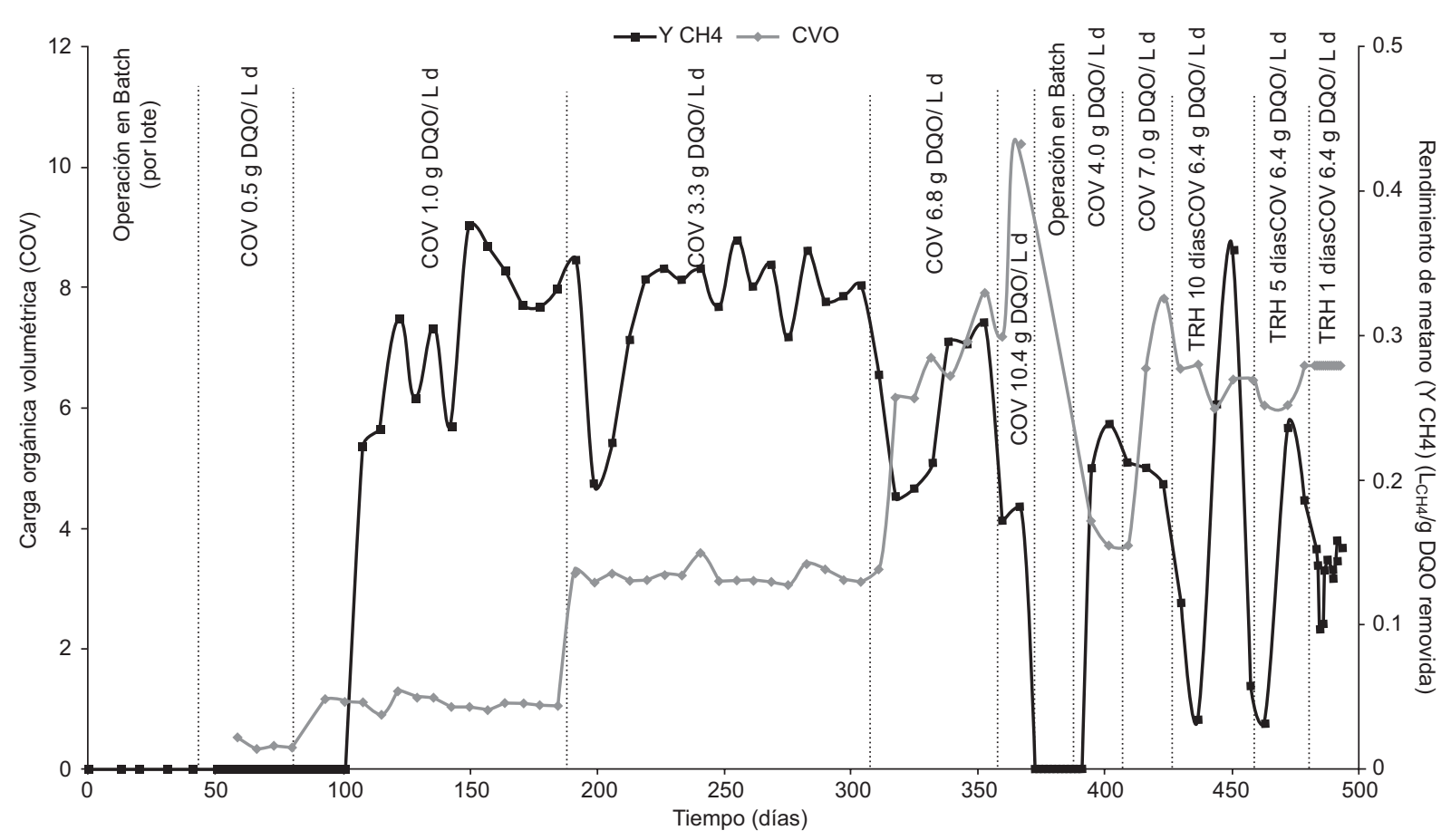

Fig. 4. Rendimiento de $\mathrm{CH}_{4}$ en el reactor de lecho fluidizado inverso anaerobio (LFI) a diferentes cargas orgánicas volumétricas. $\mathrm{DQO}=$ Demanda química de oxígeno, $\mathrm{COV}=$ Carga orgánica volumétrica, $\mathrm{TRH}=$ Tiempo de residencia hidráulica, $\mathrm{YCH}_{4}=$ Rendimiento de metano

operó con una carga de $6.4 \mathrm{~g} \mathrm{DQO} / \mathrm{L} \mathrm{d}$ y a diferentes tiempos de residencia. El mismo comportamiento observado con el incremento de cargas se manifiesta al disminuir los tiempos de residencia: al inicio de cada etapa para los diferentes TRH de 10, 5 y 1 días el rendimiento de metano es bajo y va aumentando al final de las mismas. El comportamiento del sistema al final del TRH de 1 día permite observar que la variación del rendimiento disminuye y alcanza un valor promedio de $0.1488 \mathrm{LCH}_{4} / \mathrm{g} \mathrm{DQO}$ removida . $\mathrm{El}$ análisis del comportamiento del rendimiento de metano a las diferentes cargas y TRH permite obtener información valiosa del momento en el que el sistema puede alcanzar la estabilidad. Michaud et al. (2005) y Houbron et al. (2012) indican que el rendimiento de metano, definido como la cantidad de metano producido para una cantidad dada de materia orgánica removida, es el resultado de la actividad del consorcio anaerobio de bacterias. Esto es, el valor del rendimiento de metano es constante cuando el ecosistema anaerobio usa carbono para crecer y mantenerse solamente. Al alcanzar el valor teórico del rendimiento de metano $\left(0.351 \mathrm{LCH}_{4} / \mathrm{g} \mathrm{DQO}_{\text {removida }}\right)$ podría considerarse que todo el carbono puede ser utilizado por la biopelícula para la respiración anaerobia, creciendo y manteniéndose únicamente. En el presente trabajo, en cada incremento de carga y disminución de TRH, se observa un decaimiento en el rendimiento de metano, por lo que se asume que existe una inhibición de los microorganismos en respuesta al aumento de las cargas orgánicas. Al final de cada etapa, cuando el rendimiento de metano empieza a ser estable, el carbono aportado por la vinaza cruda podría ser empleado para crecimiento y mantenimiento de la biopelícula.

En general, los resultados conseguidos durante la evaluación del desempeño del LFI en este estudio, coinciden con los anteriormente reportados (Jiménez et al. 2006) para el tratamiento de vinazas crudas (en reactores agitados de flujo continuo y utilizando vinazas con concentración de $80.5 \mathrm{~g} \mathrm{DQO} / \mathrm{L}$ ) empleando cargas orgánicas volumétricas de 1.5 a $7.5 \mathrm{~g}$ DQO/L $\mathrm{d}$, con TRH desde 53.3 días hasta 10.6 días y en donde el rendimiento de metano aumentó con incrementos de carga orgánica volumétrica hasta un valor máximo, disminuyendo posteriormente. En dicho estudio, Jiménez et al. (2006) indican que la disminución en el rendimiento de metano a determinados valores de carga orgánica volumétrica, puede ser atribuida a una inhibición de los microorganismos metanogénicos a altos valores de carga orgánica, debido a la presencia de sustancias tóxicas en las vinazas (compuestos fenólicos), así como a altas concentraciones de ácidos grasos volátiles en el agua en tratamiento. 
Cresson et al. (2006), han reportado también que al tratar vinazas en un reactor anaerobio de lecho turbulento, hubo una disminución en la eficiencia de remoción y en la actividad metanogénica específica al alcanzar una carga orgánica volumétrica de $6.0 \mathrm{~g}$ $\mathrm{DQO} / \mathrm{L} \mathrm{d}$, además encontraron una acumulación de ácidos grasos volátiles.

Lo anterior puede ser aplicado también a los resultados obtenidos en este estudio, durante el tratamiento de las vinazas a TRH de 10, 5 y 1 días, en donde al emplear vinazas ajustadas a bajas concentraciones de DQO, se asume que no hubo inhibición de los microorganismos metanogénicos debido a la posible disminución en la concentración de ácidos grasos volátiles y de los compuestos inhibitorios que están presentes en las vinazas (Zayas et al. 2010).

\section{CONCLUSIONES}

El LFI empleado en este trabajo permitió el tratamiento de vinazas crudas con características de $\mathrm{pH}$ cercanos a 4.0 y altas concentraciones de DQO de hasta $164 \mathrm{~g} \mathrm{DQO} / \mathrm{L}$ en promedio. La operación del sistema bajo una carga de $6.8 \mathrm{~g}$ DQO/L d permitió una eficiencia de remoción de DQO de $50 \%$, considerada aceptable dentro de las eficiencias alcanzadas en el tratamiento anaerobio para efluentes de alta carga.

A una carga de $6.8 \mathrm{~g} \mathrm{DQO} / \mathrm{L} \mathrm{d}$, se obtuvo un rendimiento de metano promedio de $0.2383 \mathrm{LCH}_{4} / \mathrm{g}$ $\mathrm{DQO}_{\text {removida }}$. Cuando el LFI se operó a esta carga, el tiempo en que alcanzó estabilidad fue menor que en las cargas anteriores. Por lo anterior, se considera la máxima carga de operación del sistema, ya que una carga mayor causó inestabilidad al reactor.

Para el tratamiento de vinaza cruda a cargas desde 3.3 hasta $6.4 \mathrm{~g} \mathrm{DQO} / \mathrm{Ld}$, fue necesario emplear altos valores de tiempos de residencia, desde 23 hasta 50 días. Es importante considerar que la digestión anaerobia de las vinazas crudas resulta factible e incluso atractiva desde el punto de vista energético. Sin embargo, la presencia de compuestos inhibitorios en su composición (que han sido ampliamente estudiados), puede hacer más lenta la remoción de su contenido orgánico, por lo que es necesario el empleo de altos tiempos de residencia.

La operación del LFI a diversos TRH, permitió determinar que con un TRH de 5 días, a una carga orgánica volumétrica de $6.4 \mathrm{~g} \mathrm{DQO} / \mathrm{Ld}$, se obtuvo un rendimiento de metano promedio de $0.1922 \mathrm{LCH}_{4} /$ $\mathrm{gDQO}_{\text {removida }} \mathrm{y}$ una eficiencia de remoción de DQO promedio de $57.42 \%$, alcanzando estabilidad al final del periodo experimental a este TRH.
Por otro lado, el desarrollo de la biopelícula y el rendimiento de metano constituyeron parámetros que permitieron evaluar la eficiencia del desempeño del LFI bajo diferentes cargas orgánicas volumétricas y TRH.

\section{AGRADECIMIENTOS}

Se agradece el apoyo al Proyecto de Fondos Mixtos del Estado de Veracruz, Clave 41752.

\section{REFERENCIAS}

Abdurahman N.H., Rosli Y. M. y Azhari N. H. (2013). The performance evaluation of anaerobic methods for Palm Oil Mill Effluent (POME) treatment: A review. INTECH. 4, 87-106. DOI: 10.5772/54331.

Alvarado L. A., Rustrían P. E., García A. M. A., Rodríguez J. G. C. y Houbron E. (2008). Brewery wastewater treatment using anaerobic inverse fluidized bed reactors. Bioresource Technol. 99, 3009-3015. DOI: 10.1016/j.biortech.2007.06.022.

Alvarado L. A., Sandoval R. A., Flores A. M. G., Vallejo C. N. A. y Méndez C. J. (2010). Strategies for the startup of methanogenic inverse fluidized-bed reactors using colonized particles. Water Environ. Res. 82, 387-391.

APHA (1995). Standard Methods for the examination of water and wastewater, 19 ed. American Public Health Association, Washington, D.C. EUA, 1368 pp.

Arnaiz C., Elmaleh S., Lebrato J. y Moletta R. (2005). Start- up of an anaerobic inverse turbulent bed reactor fed with wine distillery wastewater using pre-colonised bioparticles. Water Sci. Technol. 51, 153-158.

Arnaiz C., Buffiere P., Lebrato J. y Moleta R. (2007). The effect of transient changes in organic load on the performance of an anaerobic inverse turbulent bed reactor. Chem. Eng. Process. 46, 1349-1356.

DOI: 10.1016/j.cep.2006.10.017.

Beltrán de H. J., Domínguez J. R. y Partido E. (2005). Physico-chemical treatment for the depuration of wine distillery wastewaters (vinasses). Water Sci. Technol. 51, 159-166.

Choudhury S. y Sahoo A. (2012). Waste water treatment by inverse fluidization process: an overview. IJAET 3, 8-16.

COLPOS (2010). Bases técnicas para el fomento a la producción de biocombustibles en el país a partir de caña de azúcar. Colegio de Posgraduados. Servicio de Información Agroalimetaria y Pesquera. Secretaria de Agricultura, Ganadería, Desarrollo Rural, Pesca y Alimentación. Documento Informativo. Distrito Federal, México, 119 pp. 
Cresson R., Carrére H., Delgenés J. P. y Bernet N. (2006). Biofilm formation during the start-up period of an anaerobic biofilm reactor-Impact of nutrient complementation. Biochem. Eng. J. 30, 55-62. DOI: 10.1016/j.bej.2006.02.002

Fernández N., Montalvo S., Borja R., Guerrero L., Sánchez E., Cortés I., Colmenarejo M. F., Travieso L. y Raposo F. (2008). Performance evaluation of an anaerobic fluidized bed reactor with natural zeolite as support material when treating high strength distillery wastewater. Renew. Energ. 11, 2458-2466.

DOI: 10.1016/j.renene.2008.02.002

Houbron E., Alvarado L. A., Zepeda A. y Rustrián E. (2012). Methane yield and microscopic observation as monitoring biofilm behaviour parameters, during start-up phase of anaerobic inverse fluidized bed reactor. Afr. J. Biotechnol. 11, 14342-14398.

DOI: $10.5897 / \mathrm{AJB} 11.3976$

Jiménez A. M., Borja R., Martín A. y Raposo F., (2006). Kinetic analysis of the anaerobic digestion of untreated vinasses and vinasses previously treated whit Penicillium decumbens. J. Environ. Manage. 80, 303-310. DOI: 10.1016/j.jenvman.2005.09.011

Lorenzo A. Y., Doménech L. F., Eng S. F., Almazán D. O. y Chanfón C. J. M. (2015). Tratamiento industral de vinazas de destilería en reactores UASB. Revista de Tecnología Química 35, 32-45.

Michaud S., Bernet N., Buffière P. y Delgenès J.P. (2005). Use of the methane yield to indicate the metabolic behaviour of methanogenic biofilms. Process Biochem. 40, 2751-2755. DOI: 10.1016/j.procbio.2004.12.017.

Papirio S. (2012). Fluidized-bed bioreactor applications for the treatment of metal-sulfate and nitrate-contaminated mine waters. Tesis de Doctorado. Universitá degli Studi di Cassino e de Lazio Meridionale. Frosinone, Italia, $174 \mathrm{pp}$.
Sowmeyan R. y Swaminathan G. (2008). Evaluation of inverse anaerobic fluidized bed reactor for treating high streght organic waste water. Bioresource Technol. 99, 3877-3880. DOI: 10.1016/j.biortech.2007.08.021.

Thaiyalnayaki D. y Sowmeyan R. (2012). Effect of carrier materials in inverse anaerobic fluidized bed reactor for treating high strength organic waste water. J. Environment Analytic Toxicol. 2:3.

DOI: $10.4172 / 2161-0525.1000134$

Travieso L., Sánchez E., Borja R., Benítez F., Raposo F., Rincón B. y Jiménez A. M. (2006). Evaluation of a laboratory-scale stabilization pond for tertiary treatment of distillery waste previously treated by a combined anaerobic filter-aerobic trickling system. Ecol. Eng. 27, 100-108. DOI: 10.1016/j.ecoleng.2005.11.004.

Vlyssides A. G. y Barampounti E. M. (2005). Wastewater characteristics from Greek wineries and distilleries. Water Sci.Technol. 51, 53-60

Yongsheng L. (2008). Optimización de reactores biológico secuencial y de lecho fluidizado para el tratamiento de agua. Tesis de Doctorado. Universidad de Limoges. Limoges, Francia, 196 pp.

Zayas T., Romero V., Meraz M. y Salgado L. (2010). Tratamiento de agua residual con alta carga orgánica y color provenientes del proceso de vinaza. Memorias. XII Congreso Internacional de Ciencias Ambientales. Quintana Roo, México, pp. 11-14 abril. CD-ROM. 